\title{
Collision Models Can Efficiently Simulate Any Multipartite Markovian Quantum Dynamics
}

\author{
Marco Cattaneo®, ${ }^{1,2,3,{ }^{*}}$ Gabriele De Chiara $\odot,{ }^{4}$ Sabrina Maniscalco, ${ }^{3,2,5}$ Roberta Zambrini, ${ }^{1}$ and Gian Luca Giorgi ${ }^{1}$ \\ ${ }^{1}$ Instituto de Física Interdisciplinar y Sistemas Complejos (IFISC, UIB-CSIC), \\ Campus Universitat de les Illes Balears, E-07122 Palma de Mallorca, Spain \\ ${ }^{2}$ QTF Centre of Excellence, Turku Centre for Quantum Physics, Department of Physics and Astronomy, \\ University of Turku, FI-20014 Turun Yliopisto, Finland \\ ${ }^{3}$ QTF Centre of Excellence, Department of Physics, University of Helsinki, P.O. Box 43, FI-00014 Helsinki, Finland \\ ${ }^{4}$ Centre for Theoretical Atomic, Molecular and Optical Physics, Queen's University Belfast, Belfast BT7 1NN, United Kingdom \\ ${ }^{5}$ QTF Centre of Excellence, Department of Applied Physics, School of Science, Aalto University, \\ FI-00076 Aalto, Finland
}

(Received 31 October 2020; accepted 2 March 2021; published 2 April 2021)

\begin{abstract}
We introduce the multipartite collision model, defined in terms of elementary interactions between subsystems and ancillas, and show that it can simulate the Markovian dynamics of any multipartite open quantum system. We develop a method to estimate an analytical error bound for any repeated interactions model, and we use it to prove that the error of our scheme displays an optimal scaling. Finally, we provide a simple decomposition of the multipartite collision model into elementary quantum gates, and show that it is efficiently simulable on a quantum computer according to the dissipative quantum Church-Turing theorem, i.e., it requires a polynomial number of resources.
\end{abstract}

DOI: 10.1103/PhysRevLett.126.130403

Introduction.-The collision approach represents one of the most successful methods to describe the dynamics of an open quantum system, being based on the intriguing idea that enviroment-induced decoherence and dissipation arise because of rapid repeated collisions between each system unit and a set of environment ancillas, occurring during a time step $\Delta t$. This framework, whose origins can be traced back to some important works of the previous century [1-3], has given birth to a plethora of "collision" or "repeated interactions" models [4-13], which have been receiving increasing attention in recent years, especially due to their fundamental importance in the fields of quantum thermodynamics and open quantum systems. For instance, collision models have been proven useful to investigate flux rectification [14], Landauer's principle $[15,16]$, the emergence of thermalization or nonequilibrium steady states [17-26], quantum thermometry [27], quantum batteries [28], and quantum thermal machines [29-33], as well as to analyze the thermodynamics of nonthermal baths [34-36] or in the presence of strong coupling [37]. Applications outside the field of thermodynamics include the study of open quantum optical systems [38-41], simulation of non-Markovian effects $[9,11,13,42-48]$ and cascade models [8,15,49-51], quantum synchronization [52,53], entanglement generation [54-56], quantum transport [57], and quantum Darwinism [58,59].

The structure of any single-qubit collision model and the correspondence with an equivalent master equation is well understood $[6,60,61]$. In contrast, while some collision models for multipartite systems have been presented in the past few years $[8,45,54,62]$, a universal protocol suitable for efficient simulation of multipartite open system dynamics via collision models, described in terms of elementary collisions between subsystems and ancillas, has not been provided yet. Reproducing any possible open dynamics by means of elementary collision models promises to be particularly valuable to deal with the microscopic description of multipartite open systems, where global master equations are needed [69-71] and one cannot always rely on local descriptions, that may display fundamental differences, e.g., from the thermodynamic point of view [72,73]. Here, collision models are extremely useful to study the elementary exchange of heat and energy, and the microscopic production of work in each single interaction between a unit of the system and an environment ancilla [74-76]. For instance, a collision model analysis resolves the violation of the second law of thermodynamics when using a local master equation [76,77].

In this Letter, we introduce the multipartite collision model (MCM), based on elementary interactions between each unit of a multipartite system and a set of ancillary qubits of the environment. We show that the MCM is able to reproduce any Gorini-Kossakowski-Sudarshan-Lindblad (GKLS) master equation [78,79] in the limit of small time step $\Delta t \rightarrow 0^{+}$, therefore describing any possible divisible dynamical map. After providing a simple decomposition into elementary quantum gates, we prove that the MCM is efficiently simulable on a quantum computer under the 
assumptions of the dissipative quantum Church-Turing theorem [80], as it requires a number of resources that scales polynomially as a function of the number of subsystems, time, and the inverse of desired precision. This allows for the efficient simulation of a whole range of complex open quantum systems under the Markovianity assumption: by tuning our model in an intuitive way, we can mimic the effect of different types of separate and/or common baths (bosonic, fermionic, spin, etc.) at any temperature, as well as reproduce each elementary system-bath interaction characterizing a generic global master equation, with or without a nonlocal unitary system dynamics. Non-Markovian effects may be then simulated by Markovian embeddings of pseudomodes into the MCM [45]. Furthermore, by developing a method valid for any collision model, we calculate an analytical error bound for the simulation of a generic semigroup dynamics by means of the MCM, proving that its scaling is optimal.

To guarantee the generality of the MCM, we will show that it can simulate the dynamics driven by any GKLS master equation, both in the diagonal [78] and nondiagonal form [79]. The latter can be expressed by means of the Liouvillian superoperator $\mathcal{L}$ as

$$
\mathcal{L}\left[\rho_{S}(t)\right]=-i\left[\tilde{H}_{S}, \rho_{S}(t)\right]+\sum_{j, k=1}^{J} \gamma_{j k} \mathcal{D}_{F_{j}, F_{k}}\left[\rho_{S}(t)\right],
$$

where $\mathcal{D}_{O_{1}, O_{2}}[\rho]=O_{1} \rho O_{2}^{\dagger}-\frac{1}{2}\left\{O_{2}^{\dagger} O_{1}, \rho\right\}, \tilde{H}_{S}$ is an effective system Hamiltonian, $\gamma_{j k}$ is the semipositive Kossakowski matrix, while we term $\left\{F_{k}\right\}_{k=1}^{J}$ as GoriniKossakowski-Sudarshan (GKS) operators [79]. If $\mathcal{H}_{S}=$ $\bigotimes_{j=1}^{M} \mathcal{H}_{S}^{(j)}$ is the Hilbert space of the system composed of (for simplicity identical) $M$ subsystems with $\operatorname{dim}\left(\mathcal{H}_{S}^{(j)}\right)=$ $d(d<\infty)$, in general we have $J=d^{2 M}-1$. We obtain the diagonal GKLS form by diagonalizing the Kossakowski matrix through a suitable unitary matrix $C$ : we introduce the Lindblad operators [78] $L_{k}=\sum_{j=1}^{J} C_{j k} F_{j}$, and we derive the corresponding decay rates $\Gamma_{k}$ as the eigenvalues of $\gamma_{j k}$.

For the sake of clarity, we begin by assuming that each GKS operator $F_{k}$ acts nontrivially on a single subsystem only, although the MCM is not restricted to it, as we will see in the following. This assumption is satisfied by a wide range of local and global master equations [71], and corresponds to neglecting environment-mediated manybody interactions between the subsystems. Under this assumption, the total number of Lindblad operators reduces to $J=M\left(d^{2}-1\right)$, and the index $j=(m, \alpha)$ can be decomposed into two additional indexes: $m=1, \ldots, M$ labeling the subsystems and $\alpha=1, \ldots,\left(d^{2}-1\right)$ selecting the specific GKS operator acting locally thereon.

Multipartite collision model.-The procedure to implement the MCM under the assumption of local GKS (a)

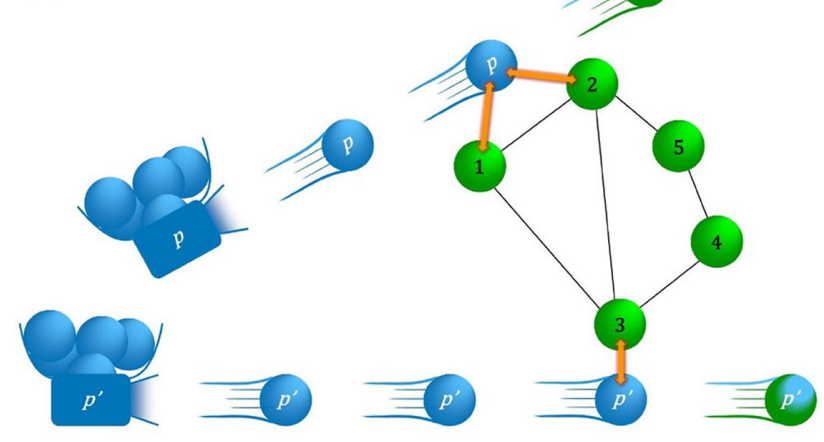

(b)

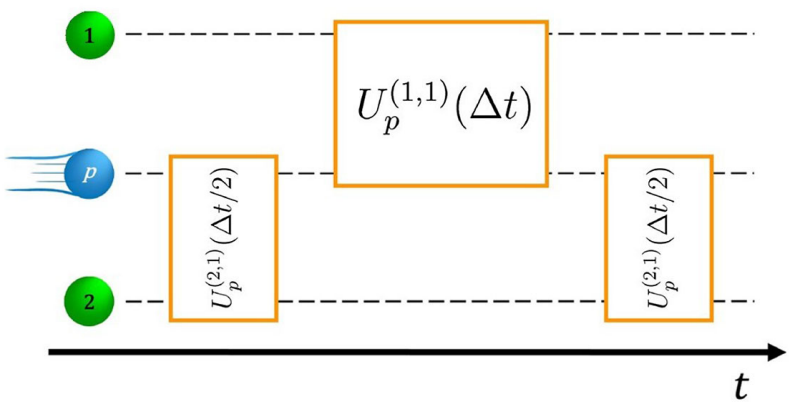

FIG. 1. (a) Pictorial representation of the MCM. The ancilla $p$ generates a term in the master equation that couples subsystems 1 and 2. The ancilla $p^{\prime}$ interacts with subsystem 3 only, and yields a local term in the master equation. (b) Circuit scheme of the interaction between ancilla $p$ and subsystems 1 and 2 . If the system is made of qubits, three two-qubit gates are required.

operators is depicted in Fig. 1. For the nondiagonal case we can identify the following five steps:

(1) For each pair of GKS operators $F_{m, \alpha}$ and $F_{m^{\prime}, \alpha^{\prime}}$ appearing in Eq. (1), consider an independent ancillary qubit of the environment labeled by $p=\left(m, \alpha, m^{\prime}, \alpha^{\prime}\right)$, and construct the sequence of local elementary subsystemancilla interactions given by

$$
U_{p}(\Delta t)=U_{p}^{(m, \alpha)}(\Delta t / 2) U_{p}^{\left(m^{\prime}, \alpha^{\prime}\right)}(\Delta t) U_{p}^{(m, \alpha)}(\Delta t / 2),
$$

where $(\hbar=1)$

$$
U_{p}^{(m, \alpha)}(\Delta t)=\exp \left(-i g_{I} \Delta t H_{I, p}^{(m, \alpha)}\right) .
$$

$H_{I, p}^{(m, \alpha)}=\left(\lambda_{p}^{(m, \alpha)} F_{m, \alpha} \sigma_{p}^{+}+\right.$H.c. $), g_{I}$ is a fixed constant with the units of energy and $\lambda_{p}^{(m, \alpha)}$ is a dimensionless parameter we can freely tune.

(2) Compose all the unitary evolutions associated to each pair of GKS operators into a global unitary operator describing the overall interaction with the environment, choosing freely the order in which we insert the former:

$$
U_{I}(\Delta t)=\prod_{p \in \mathrm{P}} U_{p}(\Delta t)
$$


where the elements of the set $\mathrm{P}$ are all the possible pairs $\left(m, \alpha, m^{\prime}, \alpha^{\prime}\right)$.

(3) Add a unitary system evolution driven by the dimensionless system Hamiltonian $H_{S}$ to obtain the final global operator for the simulation of the MCM:

$$
U_{\text {sim }}(\Delta t)=U_{S}(\Delta t) \circ U_{I}(\Delta t),
$$

with $U_{S}(\Delta t)=\exp \left(-i g_{S} \Delta t H_{S}\right)$, where $H_{S}=\tilde{H}_{S} / g_{S}$ and $g_{S}$ is a fixed constant with the units of energy, defining the order of magnitude of $\tilde{H}_{S}$.

(4) Prepare the set of environment qubits with $p \in \mathrm{P}$ in an initial separable state $\rho_{E}(0)=\bigotimes_{p \in \mathrm{P}} \eta_{p}$, where $\eta_{p}=c_{p}|\downarrow\rangle_{p}\left\langle\downarrow\left|+\left(1-c_{p}\right)\right| \uparrow\right\rangle_{p}\langle\uparrow|$, with $0 \leq c_{p} \leq 1$, is a diagonal state in the basis of $\sigma_{p}^{z}$.

(5) Apply a single step of the MCM on the system state $\rho_{S}$ as the quantum map:

$$
\phi_{\Delta t}\left[\rho_{S}\right]=\operatorname{Tr}_{E}\left[U_{\text {sim }}(\Delta t) \rho_{S} \otimes \rho_{E}(0) U_{\text {sim }}^{\dagger}(\Delta t)\right],
$$

where the trace over the environment $E$ includes the trace over each environment ancilla with $p \in \mathrm{P}$.

We show in the Supplemental Material [62] that under certain requirements the dynamics generated by the MCM corresponds to the one driven by a general GKLS master equation, Eq. (1). Specifically, we follow the standard derivation of a collision model [45]: we assume the limit of small time step $\Delta t \rightarrow 0^{+}$, with $g_{S} \ll g_{I} \ll \Delta t^{-1}$, and $\lim _{\Delta t \rightarrow 0^{+}} g_{I}^{2} \Delta t=\gamma$, where $\gamma$ is a finite energy constant. For simplicity, the coefficients $\lambda_{p}^{(m, \alpha)}$ in Eq. (3) are taken of the order of $O(1)$. Under the above assumptions, the evolution generated by a single application of the quantum map corresponds to

$$
\phi_{\Delta t}=\mathbb{I}+\Delta t \mathcal{L}+O\left(\Delta t^{2}\right),
$$

where the Liouvillian superoperator reads [62]

$$
\begin{aligned}
\mathcal{L}\left[\rho_{S}\right]= & -i\left[\tilde{H}_{S}, \rho_{S}\right]+\sum_{p \in P}\left(\gamma_{p}^{\downarrow} \mathcal{D}_{F_{m, \alpha}, F_{m^{\prime}, \alpha^{\prime}}}\left[\rho_{S}\right]\right. \\
& \left.+\gamma_{p}^{\uparrow} \mathcal{D}_{F_{m, \alpha}^{\dagger}, F_{m^{\prime}, \alpha^{\prime}}^{\dagger}}\left[\rho_{S}\right]+\text { H.c. }\right) .
\end{aligned}
$$

The coefficients are

$$
\begin{aligned}
& \gamma_{p}^{\downarrow}= \begin{cases}\gamma c_{p} \lambda_{p}^{(m, \alpha)}\left(\lambda_{p}^{\left(m^{\prime}, \alpha^{\prime}\right)}\right)^{*} & \text { if } m \neq m^{\prime} \quad \text { or } \quad \alpha \neq \alpha^{\prime} \\
\gamma \sum_{\bar{p}} c_{\bar{p}}\left|\lambda_{\bar{p}}^{(m, \alpha)}\right|^{2} & \text { otherwise }\end{cases} \\
& \gamma_{p}^{\uparrow}= \begin{cases}\gamma\left(1-c_{p}\right)\left(\lambda_{p}^{(m, \alpha)}\right)^{*} \lambda_{p}^{\left(m^{\prime}, \alpha^{\prime}\right)} & \text { if } m \neq m^{\prime} \text { or } \quad \alpha \neq \alpha^{\prime} \\
\gamma \sum_{\bar{p}}\left(1-c_{\bar{p}}\right)\left|\lambda_{\bar{p}}^{(m, \alpha)}\right|^{2} & \text { otherwise, }\end{cases}
\end{aligned}
$$

with summation over all the unordered pairs of GKS operators $\bar{p}=(m, \alpha, \bar{m}, \bar{\alpha})$. These coefficients give rise to a semipositive Kossakowski matrix, i.e., the master equation Eq. (8), is already in GKLS form. Equation (8) also contains all the terms associated with the adjoint GKS operators with Kossakowski matrix $\gamma_{p}^{\uparrow}$, that can be removed by setting $c_{p}=1 \forall p$ (i.e., by preparing each ancilla in the ground state). Given the freedom in the choice of $\lambda_{p}^{(m, \alpha)}$ and $\lambda_{p}^{\left(m^{\prime}, \alpha^{\prime}\right)}$ in the Hamiltonian of each elementary subsystemancilla interaction introduced in Eq. (3), we can engineer $\gamma_{p}^{\downarrow}$ in order to reproduce any Kossakowski matrix for the GKS operators of the master equation Eq. (8), and therefore any nondiagonal GKLS master equation Eq. (1) with effective Hamiltonian $\tilde{H}_{S}$. We can therefore conclude that repeated rapid applications of the MCM simulate the quantum semigroup dynamics driven by any Liouvillian $\mathcal{L}$ :

$$
\lim _{\Delta t \rightarrow 0^{+}}\left(\phi_{\Delta t}\right)^{n}=\exp \mathcal{L} t, \quad \text { with } \quad t=n \Delta t .
$$

This is our first major result. For a small but finite $\Delta t$, the MCM reproduces the open dynamics only for discrete times $t=n \Delta t$, where the resolution given by $\Delta t$ can be thought of as the coarse graining of the master equation [81]. Finally, it is not always necessary to take one ancilla for each pair of jump operators. In certain scenarios we may rely on a simpler version of the MCM that requires a smaller number of resources [62].

The collision scheme introduced above is particularly useful in situations where one has to apply the MCM to a symbolic GKLS master equation that cannot be diagonalized analytically. In all other cases, the MCM realizes the diagonal form of the GKLS master equation by following the same lines described above, with the prescription that we just need one ancillary qubit for each Lindblad operator $L_{k}$. Indeed, under the assumption of local GKS operators, we can write $L_{k}=\sum_{m=1}^{M} \tilde{F}_{m}^{(k)}$, where $\tilde{F}_{m}^{(k)}=$ $\sum_{\alpha=1}^{d^{2}-1} C_{\alpha k} F_{m, \alpha}$ is a local sum of GKS operators, and the evolution in Eq. (2) is replaced by the sequence of elementary interactions

$U_{k}(\Delta t)=\prod_{m=1}^{M} U_{k}^{(M-m+1)}(\Delta t / 2) \prod_{m^{\prime}=1}^{M} U_{k}^{\left(m^{\prime}\right)}(\Delta t / 2)$,

and $U_{k}^{(m)}(\Delta t)=\exp \left[-i g_{I} \Delta t\left(\lambda_{k} \tilde{F}_{m}^{(k)} \sigma_{k}^{+}+\right.\right.$H.c. $\left.)\right]$, so that $\Gamma_{k}=\lim _{\Delta t \rightarrow 0^{+}} g_{I}^{2} \Delta t\left|\lambda_{k}^{2}\right|$ is the decay rate of the $k$ th Lindblad operator. Correspondingly, the product in the global unitary operator for the interaction with the environment in Eq. (4) runs over $k=1, \ldots, J$ instead of the pairs $p \in \mathrm{P}$.

Temperature.-Note that a suitable engineering of the parameters of the MCM allows for the simulation of any thermal bath at any (even negative) temperature. For instance, to mimic a single thermal bath at temperature $T$, one can use a single ancilla prepared in a thermal state at temperature $T$, and the strength of the decay rates can be engineered by tuning the parameters $\lambda_{p}^{(m, \alpha)}$ as a function of 
$T$ [62]. Our model also allows for energy-nonconserving elementary interactions (e.g., with counterrotating terms such as $a^{\dagger} \sigma_{p}^{+}+$H.c. for a bosonic mode $a$ and a qubit ancilla labeled by $p$ ). This may generate squeezinglike terms, which corresponds to the ancillas not having the same temperature as the effective bath, and any complex scenario with multiple baths can be realized. This engineering overcomes the physical constraints of previous open system quantum simulations based on qubit ancillas [82].

Extension to many-body GKS operators and time-dependent semigroups.-The MCM also works in the case of many-body GKS operators that cannot be trivially decomposed into single subsystem-ancilla interactions (e.g., when a GKS operator is written as $F_{j}=\sigma_{1}^{-} \sigma_{2}^{+}$[83]). In this scenario, we will have an elementary collision in Eq. (3) with Hamiltonian $H_{I, p}^{(j)}=\lambda_{p}^{(j)} F_{j} \sigma_{p}^{+}+$H.c., where $F_{j}$ acts nontrivially on more than one subsystem, and therefore cannot be represented by a single two-qubit gate on a quantum computer. Its action may be implemented by multiqubit gates, as already done in quantum simulation of open systems [84], or by a decomposition in terms of two-qubit gates [85]. In general terms, we may assume to have at our disposal a set of $R$ Hamiltonians for each GKS operator $F_{j}$ (or Lindblad operator $L_{j}$ in the diagonal case), $H_{r}^{(j)}=G_{r}^{(j)} \sigma_{p}^{+}+$H.c. $\quad(p$ labels a generic ancilla $)$, that we are able to simulate by elementary multiqubit gates in our lab, through which we can build any required GKS operator Hamiltonian in Eq. (3) as $H_{I, p}^{(j)}=\sum_{r=1}^{R} \mu_{r}^{(p, j)} H_{r}^{(j)}$. Then, we can simulate the MCM by the decomposition $\exp \left(-i g_{I} \Delta t H_{I, p}^{(j)}\right)=$ $\prod_{r=1}^{R} U_{p, R-r+1}^{(j)}(\Delta t / 2) \prod_{r^{\prime}=1}^{R} U_{p, r^{\prime}}^{(j)}(\Delta t / 2)+O\left(g_{I}^{3} \Delta t^{3}\right)$, with $U_{p, r}^{(j)}(\Delta t)=\exp \left(-i g_{I} \Delta t \mu_{r}^{(p, j)} H_{r}^{(j)}\right)$, which still brings an error of the order of $O\left(\Delta t^{2}\right)$ in Eq. (7) [62]. Note that, if we go back to the condition of local GKS operators, for simplicity we can assume to be able to directly implement any elementary gate $U_{p}^{(m, \alpha)}$ in the lab, and therefore for the nondiagonal case $R=1$. In the diagonal case, we can interpret $R$ as the number of different elementary subsystem-ancilla interactions in Eq. (11), therefore $R=M$. Finally, extensions to time-dependent semigroups in which the Kossakowski matrix in Eq. (1) depends on time, $\gamma_{j k}(t)$ semipositive for any time $t$, are immediate: we just need to set a time-dependent parameter $\lambda_{p}^{(m, \alpha)}(t)$ in the Hamiltonian of Eq. (3), and to make it vary as a function of $t$. Analogously, we can make the system Hamiltonian depend on time as well, as $H_{S}(t)$.

Error estimation.-Previous treatments of the error analysis for a collision model have usually neglected higher-order terms in the Taylor expansion, e.g., see the detailed discussion in Ref. [12]. Sometimes this may not be accurate, since the infinite series of higher-order terms may bring a non-negligible contribution [86]. Here, we estimate an analytical error bound for the MCM by keeping all the terms of the infinite Taylor expansion through a method based on Suzuki's higher-order integrators [87] which can be found in the Supplemental Material [62], whose validity applies to any collision model. For the sake of a general description, we compute the error bound without assuming the GKS operators locality, and therefore relying on the sets of $R$ many-body Hamiltonians $H_{r}^{(j)}$ introduced above.

To estimate the error bound we employ the $1 \rightarrow 1$ superoperator norm $\|\mathcal{T}\|_{1 \rightarrow 1}$ and the operator norm $\|A\|_{\infty}$ [88]. We can identify four different kinds of error made by approximating the semigroup evolution through the collision model: Global error: $\epsilon_{g}=\left\|\exp \mathcal{L} t-\left(\phi_{\Delta t}\right)^{n}\right\|_{1 \rightarrow 1}$, with $\quad \Delta t=t / n ; \quad$ Singlestep error: $\quad \epsilon_{s}=\left\|\exp \mathcal{L} \Delta t-\phi_{\Delta t}\right\|_{1 \rightarrow 1} ; \quad$ Truncation error: $\epsilon_{t}=\|\exp \mathcal{L} \Delta t-(\mathbb{I}+\Delta t \mathcal{L})\|_{1 \rightarrow 1}$; Collision error: $\epsilon_{c}=\left\|\phi_{\Delta t}-(\mathbb{I}+\Delta t \mathcal{L})\right\|_{1 \rightarrow 1}$.

Following Lemma 2 in Ref. [90], we have $\epsilon_{g} \leq n \epsilon_{s}$, and according to the triangle inequality $\epsilon_{s} \leq \epsilon_{t}+\epsilon_{c}$. The latter errors can be bound as [62]

$\epsilon_{t} \leq 2 e(R \Lambda(1+J R \Lambda) \Delta t)^{2}, \quad 2 R \Lambda(1+J R \Lambda) \Delta t<1$,

$$
\epsilon_{c} \leq \operatorname{pol}_{1}\left(\Lambda, \Xi, g_{S}, \gamma\right) \Delta t^{2}+\operatorname{pol}_{2}\left(\Lambda, \Xi, g_{S}, \gamma\right) \Delta t^{3},
$$

where $\mathrm{pol}_{1}$ and $\mathrm{pol}_{2}$ are polynomial functions of $g_{S}$, $\gamma, \Lambda=\max _{r, j, p}\left(\left\|H_{S}\right\|_{\infty},\left\|\mu_{r}^{(p, j)} H_{r}^{(j)}\right\|_{\infty}\right)$ and $\Xi$, equal to the total number of different elementary unitary evolutions driven by a single $H_{r}^{(j)}$ in Eq. (4) (in the case of the MCM for the diagonal master equation, we have $\Xi=R J$, for the nondiagonal scenario $\Xi=R|\mathrm{P}|$ ). The exact expressions of $\mathrm{pol}_{1}$ and $\mathrm{pol}_{2}$, as well as the above bounds under the assumption of $k$ locality [80], are discussed with further details in the Supplemental Material [62]. Here, we just remark that the global error of the MCM follows the behavior

$$
\epsilon_{g}=O\left(n \Delta t^{2}\right)=O\left(t^{2} / n\right) .
$$

This scaling is optimal for the error made by simulating an open system dynamics via a general scheme of repeated unitary evolutions [91], and therefore via general collision models. Such scaling, for instance, is always saturated by the truncation error $\epsilon_{t}$, which is the same for any model of rapid repeated interactions. This is our second major result.

Resource estimation for quantum simulation.-To address the quantum simulation efficiency of the MCM we assume the $k$ locality of the Liouvillian $\mathcal{L}$, namely, that it can be written as a sum of Liouvillians $\mathcal{L}_{\sigma}$ nontrivially acting on $k$ subsystems only: $\mathcal{L}=\sum_{\sigma=1}^{K} \mathcal{L}_{\sigma}$. This is a standard assumption for quantum simulation on a circuital quantum computer, introduced by Kliesch et al. for open systems [80,92], and first imposed in the seminal paper by 
Lloyd on Hamiltonian quantum simulation [93]. $K \leq M^{k}$ is the total number of possible $k$-local terms, that for large $M$ goes as $K \sim M^{k} /\left(k ! e^{k}\right)$ [62]. We estimate the number of resources focusing on the MCM for the diagonal GKLS master equation only, given that this is certainly the most convenient scheme for the simulation on a quantum computer. We allow for many-body GKS operators, and we count the number of elementary gates driven by the sets of $R$ Hamiltonians $\left\{H_{r}^{(\sigma, j)}\right\}_{r=1}^{R}$, corresponding to the Lindblad operators $L_{\sigma, j}$ of each $\mathcal{L}_{\sigma}$. Note that $k$ locality implies $R<d^{2 k}$. Under these assumptions and with $H_{S}=\sum_{\sigma=1}^{K} H_{S}^{(\sigma)}$, the error bound in Eq. (13) is conveniently rewritten by substituting $\Lambda \rightarrow \Lambda^{\prime}=\max _{r, j, \sigma}\left(\left\|H_{S}^{(\sigma)}\right\|_{\infty},\left\|\mu_{r}^{(\sigma, j)} H_{r}^{(\sigma, j)}\right\|_{\infty}\right), \quad \Xi \rightarrow \Xi^{\prime}=$ $K R J_{k}$ [62], where the total number of Lindblad operators for $k$-local Liouvillians is bound by $J_{k} \leq d^{2 k}-1$. $\Lambda^{\prime}$ does not increase with the total number of subsystems, while $\Xi^{\prime}$ scales polynomially with $M$. Moreover, the bound in Eq. (12) is multiplied by $K^{2}$ and modified with $\Lambda \rightarrow \Lambda^{\prime}$, $J \rightarrow J_{k}$ as above, thus it scales polynomially with $M$. Therefore, we can set $\epsilon_{g} \leq f(M) t^{2} / n$, where $f(M)$ is a polynomial function of the total number of subsystems [62].

For a single time step of the MCM, we need one ancilla for each Lindblad operator of each $k$-local Liouvillian. Therefore, we require $K J_{k}$ ancillas per time step. For the simulation up to time $t$ within a global precision of $\epsilon_{g}$, we need $N_{A}=\left\lceil K J_{k} f(M) t^{2} / \epsilon_{g}\right\rceil$ ancillas, which is a polynomial function poly $\left(M, t, 1 / \epsilon_{g}\right)$ and therefore provides us with an efficient number of ancillas [94] for quantum simulation $[80,89]$.

We need $2 R-1$ elementary quantum gates for each Lindblad operator of a single time step. Hence, the total number of gates in a single time step is $(2 R-1) K J_{k}+N_{G}^{(S)}$, where $N_{G}^{(S)}$ is the necessary number of gates to simulate the free system evolution $U_{S}(\Delta t)$ in Eq. (5), which is efficient under the required assumptions [93]. Consequently, to simulate the dynamics up to time $t$ making an error not bigger than $\epsilon_{g}$, we need

$$
N_{G}=\left\lceil\left[(2 R-1) K J_{k}+N_{G}^{(S)}\right] f(M) t^{2} / \epsilon_{g}\right\rceil
$$

gates. Under the condition of local GKS operators, we can substitute $R=k$ in Eq. (15). Once again, $N_{G}=\operatorname{poly}\left(M, t, 1 / \epsilon_{g}\right)$ and therefore the $\mathrm{MCM}$ is efficiently simulable on a quantum computer according to the dissipative quantum Church-Turing theorem. This is our third major result. The total number of gates scales as $t^{2} / \epsilon_{g}$, which is optimal [91] for collision models.

Conclusions. - We have presented the multipartite collision model (MCM), able to reproduce any Markovian dynamics (or, more precisely, any divisible dynamical map) of a general system made of $M$ subsystems by means of elementary interactions between each subsystem and a single environment ancilla, which can be efficiently simulated through elementary quantum gates. Furthermore, we have derived an analytical error bound for the simulation of generic semigroup dynamics via the MCM, and observed that it displays an optimal scaling. In light of the above findings, we believe that the MCM will play a major role in the study and simulation of multipartite open quantum systems in the future.

Our results pave the way towards general applications of the collision approach to global master equations, manybody dissipative collective effects like superradiance or synchronization, transport in complex open systems, as well as to a wide range of problems in quantum thermodynamics, such as the study of Landauer's principle in any multipartite system, of composed thermal machines or of the microscopic exchange of energy between subsystems and ancillas. Finally, the efficient simulation of the MCM on a noisy intermediate-scale quantum device is within our reach through currently available technology [95].

M. C. thanks Rodrigo Martínez-Peña for useful suggestions. This work was supported by CAIB through QUAREC project (PRD2018/47), by the Spanish State Research Agency through projects PID2019-109094 GBC21, and through the Severo Ochoa and María de Maeztu Program for Centers and Units of Excellence in R\&D (MDM-2017-0711). G. L. G. is funded by the Spanish Ministerio de Educación y Formación Profesional/ Ministerio de Universidades and co-funded by the University of the Balearic Islands through the Beatriz Galindo program (BG20/00085). G. D. C. acknowledges support from the UK EPSRC grants No. EP/S02994X/1 and No. EP/T026715/1. S. M. acknowledges financial support from the Academy of Finland via the Centre of Excellence program (Project No. 336814).

*marcocattaneo@ifisc.uib-csic.es

[1] R. Karplus and J. Schwinger, A note on saturation in microwave spectroscopy, Phys. Rev. 73, 1020 (1948).

[2] J. Rau, Relaxation Phenomena in spin and harmonic oscillator systems, Phys. Rev. 129, 1880 (1963).

[3] R. Dümcke, The low density limit for an N-level system interacting with a free bose or fermi gas, Commun. Math. Phys. 97, 331 (1985).

[4] V. Scarani, M. Ziman, P. Śtelmachovič, N. Gisin, and V. Bužek, Thermalizing Quantum Machines: Dissipation and Entanglement, Phys. Rev. Lett. 88, 097905 (2002).

[5] M. Ziman, P. Štelmachovič, V. Bužek, M. Hillery, V. Scarani, and N. Gisin, Diluting quantum information: An analysis of information transfer in system-reservoir interactions, Phys. Rev. A 65, 042105 (2002).

[6] M. Ziman and V. Bužek, All (qubit) decoherences: Complete characterization and physical implementation, Phys. Rev. A 72, 022110 (2005).

[7] S. Attal and Y. Pautrat, From repeated to continuous quantum interactions, Ann. Henri Poincaré 7, 59 (2006). 
[8] V. Giovannetti and G. M. Palma, Master Equations for Correlated Quantum Channels, Phys. Rev. Lett. 108, 040401 (2012).

[9] F. Ciccarello, G. M. Palma, and V. Giovannetti, Collisionmodel-based approach to non-Markovian quantum dynamics, Phys. Rev. A 87, 040103 (2013).

[10] L. Bruneau, A. Joye, and M. Merkli, Repeated interactions in open quantum systems, J. Math. Phys. (N.Y.) 55, 075204 (2014).

[11] B. Vacchini, General structure of quantum collisional models, Int. J. Quantum. Inform. 12, 1461011 (2014).

[12] D. Grimmer, D. Layden, R. B. Mann, and E. MartínMartínez, Open dynamics under rapid repeated interaction, Phys. Rev. A 94, 032126 (2016).

[13] S. Kretschmer, K. Luoma, and W. T. Strunz, Collision model for non-Markovian quantum dynamics, Phys. Rev. A 94, 012106 (2016).

[14] G. T. Landi, E. Novais, M. J. de Oliveira, and D. Karevski, Flux rectification in the quantum XXZ chain, Phys. Rev. E 90, 042142 (2014).

[15] S. Lorenzo, R. McCloskey, F. Ciccarello, M. Paternostro, and G. M. Palma, Landauer's Principle in Multipartite Open Quantum System Dynamics, Phys. Rev. Lett. 115, 120403 (2015).

[16] M. Pezzutto, M. Paternostro, and Y. Omar, Implications of non-markovian quantum dynamics for the landauer bound, New J. Phys. 18, 123018 (2016).

[17] D. Karevski and T. Platini, Quantum Nonequilibrium Steady States Induced by Repeated Interactions, Phys. Rev. Lett. 102, 207207 (2009).

[18] F. Barra and C. Lledó, Stochastic thermodynamics of quantum maps with and without equilibrium, Phys. Rev. E 96, 052114 (2017).

[19] M. Lostaglio, Á. M. Alhambra, and C. Perry, Elementary thermal operations, Quantum 2, 52 (2018).

[20] S. Cusumano, V. Cavina, M. Keck, A. De Pasquale, and V. Giovannetti, Entropy production and asymptotic factorization via thermalization: A collisional model approach, Phys. Rev. A 98, 032119 (2018).

[21] S. Seah, S. Nimmrichter, and V. Scarani, Nonequilibrium dynamics with finite-time repeated interactions, Phys. Rev. E 99, 042103 (2019).

[22] O. Arisoy, S. Campbell, and Ö. E. Müstecapliolu, Thermalization of finite many-body systems by a collision model, Entropy 21, 1182 (2019).

[23] A. Manatuly, W. Niedenzu, R. Román-Ancheyta, B. Çakmak, Ö. E. Müstecaplolu, and G. Kurizki, Collectively enhanced thermalization via multiqubit collisions, Phys. Rev. E 99, 042145 (2019).

[24] K. Korzekwa and M. Lostaglio, Quantum advantage in simulating stochastic processes, arXiv:2005.02403.

[25] J. Ehrich, M. Esposito, F. Barra, and J. M. Parrondo, Microreversibility and thermalization with collisional baths, Physica (Amsterdam) 552A, 122108 (2020).

[26] G. Guarnieri, D. Morrone, B. Çakmak, F. Plastina, and S. Campbell, Non-equilibrium steady-states of memoryless quantum collision models, Phys. Lett. A 384, 126576 (2020).

[27] S. Seah, S. Nimmrichter, D. Grimmer, J. P. Santos, V. Scarani, and G. T. Landi, Collisional Quantum Thermometry, Phys. Rev. Lett. 123, 180602 (2019).
[28] F. Barra, Dissipative Charging of a Quantum Battery, Phys. Rev. Lett. 122, 210601 (2019).

[29] C. Da, W. Niedenzu, Ö. Müstecaplolu, and G. Kurizki, Multiatom Quantum coherences in micromasers as fuel for thermal and nonthermal machines, Entropy 18, 244 (2016).

[30] M. Pezzutto, M. Paternostro, and Y. Omar, An out-ofequilibrium non-Markovian quantum heat engine, Quantum Sci. Technol. 4, 025002 (2019).

[31] A. Hewgill, J. O. González, J. P. Palao, D. Alonso, A. Ferraro, and G. De Chiara, Three-qubit refrigerator with two-body interactions, Phys. Rev. E 101, 012109 (2020).

[32] G. De Chiara and M. Antezza, Quantum machines powered by correlated baths, Phys. Rev. Research 2, 033315 (2020).

[33] N. Piccione, G. De Chiara, and B. Bellomo, Quantum thermal cycles based on non-resonant exchange interactions, arXiv:2010.03489.

[34] G. Manzano, F. Galve, R. Zambrini, and J. M. R. Parrondo, Entropy production and thermodynamic power of the squeezed thermal reservoir, Phys. Rev. E 93, 052120 (2016).

[35] G. Manzano, Squeezed thermal reservoir as a generalized equilibrium reservoir, Phys. Rev. E 98, 042123 (2018).

[36] F. L. S. Rodrigues, G. De Chiara, M. Paternostro, and G. T. Landi, Thermodynamics of Weakly Coherent Collisional Models, Phys. Rev. Lett. 123, 140601 (2019).

[37] P. Strasberg, Repeated Interactions and Quantum Stochastic Thermodynamics at Strong Coupling, Phys. Rev. Lett. 123, 180604 (2019).

[38] L. Bruneau, Mixing properties of the one-atom maser, J. Stat. Phys. 155, 888 (2014).

[39] F. Ciccarello, Collision models in quantum optics, Quantum Meas. Quantum Metrol. 4, 53 (2017).

[40] D. Cilluffo, A. Carollo, S. Lorenzo, J. A. Gross, G. M. Palma, and F. Ciccarello, Collisional picture of quantum optics with giant emitters, Phys. Rev. Research 2, 043070 (2020).

[41] A. Carollo, D. Cilluffo, and F. Ciccarello, Mechanism of decoherence-free coupling between giant atoms, Phys. Rev. Research 2, 043184 (2020).

[42] N. K. Bernardes, A. R. R. Carvalho, C. H. Monken, and M. F. Santos, Environmental correlations and Markovian to nonMarkovian transitions in collisional models, Phys. Rev. A 90, 032111 (2014).

[43] R. McCloskey and M. Paternostro, Non-Markovianity and system-environment correlations in a microscopic collision model, Phys. Rev. A 89, 052120 (2014).

[44] B. Çakmak, M. Pezzutto, M. Paternostro, and Ö. E. Müstecaplolu, Non-Markovianity, coherence, and systemenvironment correlations in a long-range collision model, Phys. Rev. A 96, 022109 (2017).

[45] S. Lorenzo, F. Ciccarello, and G. M. Palma, Composite quantum collision models, Phys. Rev. A 96, 032107 (2017).

[46] S. Campbell, F. Ciccarello, G. M. Palma, and B. Vacchini, System-environment correlations and Markovian embedding of quantum non-Markovian dynamics, Phys. Rev. A 98, 012142 (2018).

[47] J. Jin and C.-s. Yu, Non-Markovianity in the collision model with environmental block, New J. Phys. 20, 053026 (2018).

[48] Z.-X. Man, Y.-J. Xia, and R. Lo Franco, Temperature effects on quantum non-Markovianity via collision models, Phys. Rev. A 97, 062104 (2018). 
[49] S. Lorenzo, A. Farace, F. Ciccarello, G. M. Palma, and V. Giovannetti, Heat flux and quantum correlations in dissipative cascaded systems, Phys. Rev. A 91, 022121 (2015).

[50] S. Cusumano, A. Mari, and V. Giovannetti, Interferometric quantum cascade systems, Phys. Rev. A 95, 053838 (2017).

[51] S. Cusumano, A. Mari, and V. Giovannetti, Interferometric modulation of quantum cascade interactions, Phys. Rev. A 97, 053811 (2018).

[52] G. Karpat, I. Yalçlnkaya, and B. Çakmak, Quantum synchronization in a collision model, Phys. Rev. A 100, 012133 (2019).

[53] G. Karpat, skender Yalnkaya, B. akmak, G. L. Giorgi, and R. Zambrini, Synchronization and non-markovianity in open quantum systems, arXiv:2008.03310.

[54] S. Daryanoosh, B. Q. Baragiola, T. Guff, and A. Gilchrist, Quantum master equations for entangled qubit environments, Phys. Rev. A 98, 062104 (2018).

[55] B. Çakmak, S. Campbell, B. Vacchini, Ö. E. Müstecaplolu, and M. Paternostro, Robust multipartite entanglement generation via a collision model, Phys. Rev. A 99, 012319 (2019).

[56] X.-M. Li, Y.-X. Chen, Y.-J. Xia, Q. Zhang, and Z.-X. Man, Quantum entanglement dynamics based on composite quantum collision model, Chin. Phys. B 29, 060302 (2020).

[57] D. A. Chisholm, G. García-Pérez, M. A. C. Rossi, G. M. Palma, and S. Maniscalco, Stochastic collision model approach to transport phenomena in quantum networks, arXiv:2010.05618.

[58] S. Campbell, B. Çakmak, Ö. E. Müstecaplolu, M. Paternostro, and B. Vacchini, Collisional unfolding of quantum Darwinism, Phys. Rev. A 99, 042103 (2019).

[59] G. García-Pérez, D. A. Chisholm, M. A. C. Rossi, G. M. Palma, and S. Maniscalco, Decoherence without entanglement and quantum Darwinism, Phys. Rev. Research 2, 012061(R) (2020).

[60] T. Rybár, S. N. Filippov, M. Ziman, and V. Bužek, Simulation of indivisible qubit channels in collision models, J. Phys. B 45, 154006 (2012).

[61] S. N. Filippov, J. Piilo, S. Maniscalco, and M. Ziman, Divisibility of quantum dynamical maps and collision models, Phys. Rev. A 96, 032111 (2017).

[62] See Supplemental Material at http://link.aps.org/ supplemental/10.1103/PhysRevLett.126.130403 for a brief review of further multipartite models, some examples, and the derivation of all the results, which includes Refs. [63-68].

[63] N. Hatano and M. Suzuki, Finding exponential product formulas of higher orders, in Quantum Annealing and Other Optimization Methods (Springer, New York, 2005), pp. 37-68.

[64] M. Cattaneo, G. L. Giorgi, S. Maniscalco, and R. Zambrini, Symmetry and block structure of the Liouvillian superoperator in partial secular approximation, Phys. Rev. A 101, 042108 (2020).

[65] A. M. Childs, D. Maslov, Y. Nam, N. J. Ross, and Y. Su, Toward the first quantum simulation with quantum speedup - Supporting information, Proc. Natl. Acad. Sci. U.S.A. 115, 9456 (2018).
[66] D. W. Berry, G. Ahokas, R. Cleve, and B. C. Sanders, Efficient quantum algorithms for simulating sparse Hamiltonians, Commun. Math. Phys. 270, 359 (2007).

[67] A. M. Childs and T. Li, Efficient simulation of sparse Markovian quantum dynamics, Quantum Inf. Comput. 17, 901 (2017).

[68] D. A. Lidar, P. Zanardi, and K. Khodjasteh, Distance bounds on quantum dynamics, Phys. Rev. A 78, 012308 (2008).

[69] P. P. Hofer, M. Perarnau-Llobet, L. D. M. Miranda, G. Haack, R. Silva, J. B. Brask, and N. Brunner, Markovian master equations for quantum thermal machines: Local versus global approach, New J. Phys. 19, 123037 (2017).

[70] J. O. González, L. A. Correa, G. Nocerino, J. P. Palao, D. Alonso, and G. Adesso, Testing the validity of the local' and global' GKLS master equations on an exactly solvable model, Open Syst. Inf. Dyn. 24, 1740010 (2017).

[71] M. Cattaneo, G. L. Giorgi, S. Maniscalco, and R. Zambrini, Local versus global master equation with common and separate baths: superiority of the global approach in partial secular approximation, New J. Phys. 21, 113045 (2019).

[72] A. Levy and R. Kosloff, The Local Approach to Quantum Transport May Violate the Second Law of Thermodynamics, Europhys. Lett. 107, 20004 (2014).

[73] J. T. Stockburger and T. Motz, Thermodynamic deficiencies of some simple Lindblad operators, Fortschr. Phys. 65, 1600067 (2017).

[74] F. Barra, The thermodynamic cost of driving quantum systems by their boundaries, Sci. Rep. 5, 14873 (2015).

[75] P. Strasberg, G. Schaller, T. Brandes, and M. Esposito, Quantum and Information Thermodynamics: A Unifying Framework Based on Repeated Interactions, Phys. Rev. X 7, 021003 (2017).

[76] G. De Chiara, G. T. Landi, A. Hewgill, B. Reid, A. Ferraro, A. J. Roncaglia, and M. Antezza, Reconciliation of quantum local master equations with thermodynamics, New J. Phys. 20, 113024 (2018).

[77] A. Hewgill, G. De Chiara, and A. Imparato, Quantum thermodynamically consistent local master equations, Phys. Rev. Research 3, 013165 (2020).

[78] G. Lindblad, On the generators of quantum dynamical semigroup, Commun. Math. Phys. 48, 119 (1976).

[79] V. Gorini, A. Kossakowski, and E. C. G. Sudarshan, Completely positive dynamical semigroups of N-level systems, J. Math. Phys. (N.Y.) 17, 821 (1976).

[80] M. Kliesch, T. Barthel, C. Gogolin, M. Kastoryano, and J. Eisert, Dissipative Quantum Church-Turing Theorem, Phys. Rev. Lett. 107, 120501 (2011).

[81] D. Farina and V. Giovannetti, Open-quantum-system dynamics: Recovering positivity of the Redfield equation via the partial secular approximation, Phys. Rev. A 100, 012107 (2019).

[82] H. Wang, S. Ashhab, and F. Nori, Quantum algorithm for simulating the dynamics of an open quantum system, Phys. Rev. A 83, 062317 (2011).

[83] G. Manzano, G. L. Giorgi, R. Fazio, and R. Zambrini, Boosting the performance of small autonomous refrigerators via common environmental effects, New J. Phys. 21, 123026 (2019).

[84] J. T. Barreiro, M. Müller, P. Schindler, D. Nigg, T. Monz, M. Chwalla, M. Hennrich, C. F. Roos, P. Zoller, and R. Blatt, 
An open-system quantum simulator with trapped ions, Nature (London) 470, 486 (2011).

[85] M. A. Nielsen and I. Chuang, Quantum Computation and Quantum Information: 10th Anniversary Edition (Cambridge University Press, Cambridge, England, 2010).

[86] A. M. Childs, Y. Su, M. C. Tran, N. Wiebe, and S. Zhu, Theory of Trotter Error with Commutator Scaling, Phys. Rev. X 11, 011020 (2021).

[87] M. Suzuki, Decomposition formulas of exponential operators and lie exponentials with some applications to quantum mechanics and statistical physics, J. Math. Phys. (N.Y.) 26, 601 (1985).

[88] The superoperator norm is defined as [80,89] $\|\mathcal{T}\|_{1 \rightarrow 1}=\sup _{\|A\|_{1}=1}\|\mathcal{T}[A]\|_{1}$, where $\|A\|_{1}=\operatorname{Tr}\left[\sqrt{A^{\dagger} A}\right]$ is the trace norm. The operator or infinity norm is defined as $\|A\|_{\infty}=\sup _{\|v\|=1}\|A v\|$, where $\|v\|$ is the standard vector norm.

[89] R. Sweke, I. Sinayskiy, D. Bernard, and F. Petruccione, Universal simulation of Markovian open quantum systems, Phys. Rev. A 91, 062308 (2015).
[90] R. Sweke, I. Sinayskiy, and F. Petruccione, Simulation of singlequbit open quantum systems, Phys. Rev. A 90, 022331 (2014).

[91] R. Cleve and C. Wang, Efficient quantum algorithms for simulating lindblad evolution, in 44th International Colloquium on Automata, Languages, and Programming (ICALP 2017) (Schloss Dagstuhl - Leibniz-Zentrum für Informatik, Wadern, 2017), pp. 17:1-17:14.

[92] T. Barthel and M. Kliesch, Quasilocality and Efficient Simulation of Markovian Quantum Dynamics, Phys. Rev. Lett. 108, 230504 (2012).

[93] S. Lloyd, Universal quantum simulators, Science 273, 1073 (1996).

[94] We are assuming to use a new set of ancillas at each time step. In case of not having at our disposal a large number of available ancillas, one may also reinitialize the set of $K J_{k}$ ancillas to the state $\rho_{E}(0)$ before each time step, and the results of this work would still hold.

[95] G. García-Pérez, M. A. C. Rossi, and S. Maniscalco, IBM Q experience as a versatile experimental testbed for simulating open quantum systems, npj Quantum Inf. 6, 1 (2020). 